\title{
Clinico-Dermoscopic Correlation in Nail Sarcoidosis
}

\author{
Maouni Safae'*, El Anzi Ouiam¹, Sqalli Asmae, Zenati Kaoutar², Meziane Mariam', Benzekri Leila ${ }^{1}$, Senouci \\ Karima $^{1}$ and Hassam Badredine ${ }^{1}$
}

${ }^{1}$ Department of Dermatology-Venereology, Rabat, Morocco

${ }^{2}$ Department of Anatomopathology, Morocco

*Corresponding author: Maouni Safae, Department of Dermatology-Venereology, Morocco

\begin{abstract}
Sarcoidosis is a granulomatous systemic disease of undetermined origin. Its dermatological manifestations are present in $25 \%$ of cases and they are very polymorphic. Nail abnormalities are unusual in sarcoidosis and are often an indication of systemic involvement. Dermoscopy of the nail may be very useful in some isolated cases and can guide us to the right diagnosis. We report a rare case of systemic sarcoidosis presenting as nail dystrophy with painful phalanges and underlying bony cysts, treated successfully by oral corticosteroid with good evolution.
\end{abstract}

Keywords: Sarcoidosis; Nail; Systemic involvement; Dermoscopy

\section{Introduction}

Sarcoidosis is a multisystem granulomatous disease that can affect all organs of the body. The disorder course can vary from a self-limiting illness to a chronic disease with flares and relapses. Nail changes are rare in sarcoidosis and they are frequently an indication of systemic disease. We report a rare case of systemic sarcoidosis presenting as nail dystrophy with painful phalanges and underlying bony cysts.

\section{Observation}

A 57-year-old Moroccan woman, without medical history, was admitted to the dermatology department with two-year history of painful toe nail lesions. Physical examination showed a paronychia of all toes affected with thickening of the plate, hyperkeratosis and splinter hemorrhages, associated to a longitudinal nail fracture with localized groove longitudinal nail plate deformity of the right big toe and hyperkeratotic verrucous lesions of the nail body and proximal nail fold of the second toes (Figure 1). Dermoscopic evaluation of proximal nail fold revealed a pink coloration of the background, change of the microvascular architecture, linear vessels of different diameter, glomerular and serpiginous vessels, large areas of white scar-like depigmentation, translucent orange globular-like structures and white scale (Figure 2). Dermoscopy of nail plate showed a nail thickening, subungual hyperkeratosis, Splinter hemorrhages, dark dots, nonparallel lesion edg, yellow or white nail plate and longitudinal parallel white lines (Figure 2). Mycological examination of the nail was negative. A toes X-ray demonstrated bony cysts, trabecular pattern and osteolysis in distal phalanx. CT showed multiples lymphadenopathy above and under diaphragmatic. A punch biopsy taken from the nail matrix, the lymphadenopathy and salivary gland showed sarcoid granulomas in the dermis and a chronic inflammatory infiltrate (Figure 3). the serum level of the angiotensin- converting enzyme was normal (10$70 \mu \mathrm{g} / \mathrm{L})$. With all this finding, the diagnosis of systemic sarcoidosis was making, and the patient receive a systemic treatment with corticosteroid: $0.5 \mathrm{mg} / \mathrm{kg} /$ day. After 1 week of treatment, the patient had a good response. 


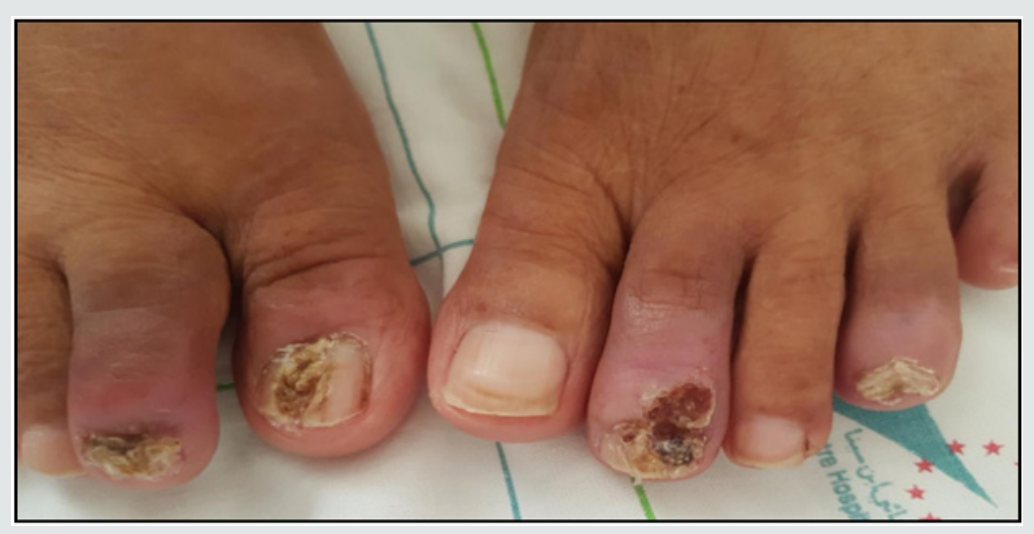

Figure 1: Nail sarcoidosis

Paronychia of all toes affected, thickening of the plate, hyperkeratosis and splinter hemorrhages, longitudinal nail fracture of the right big toe, hyperkeratotic verrucous lesions of the nail body and proximal nail fold of the second toes.

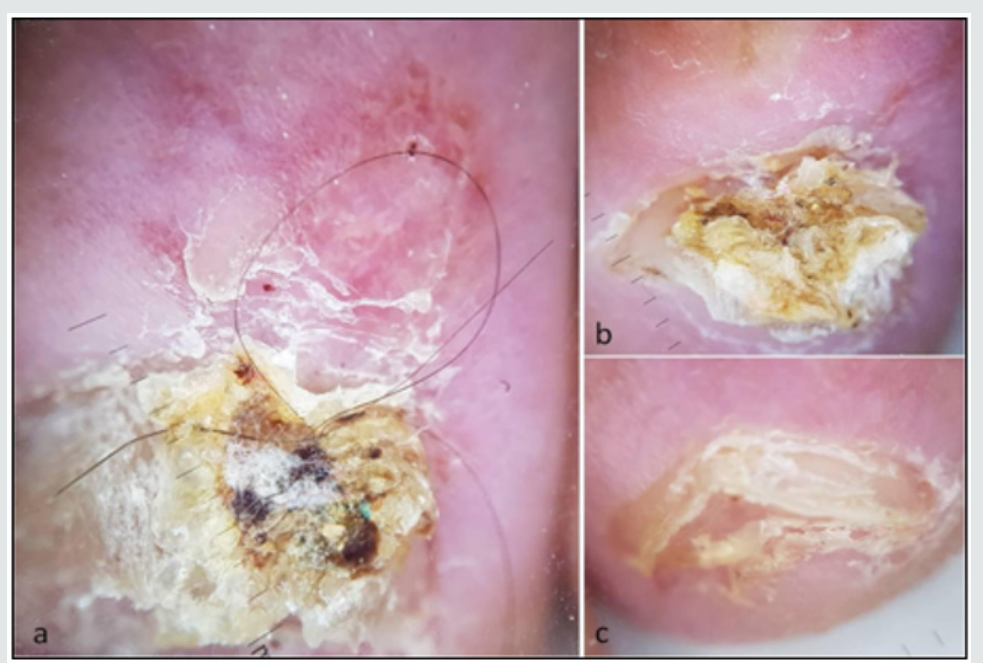

Figure 2: Dermoscopy of nail sarcoidosis

a. Dermoscopy of proximal nail fold showing pink coloration of the background, linear vessels of different diameter with splinter hemorrhages, large areas of white scar-like depigmentation, translucent orange globular-like structures and white scale.

b. Dermoscopy of nail plate showing a nail thickening, hyperkeratosis and yellow nail plate with glomerular vessels in the proximal nail fold.

c. Nail edge dermoscopy showing subungual hyperkeratosis.

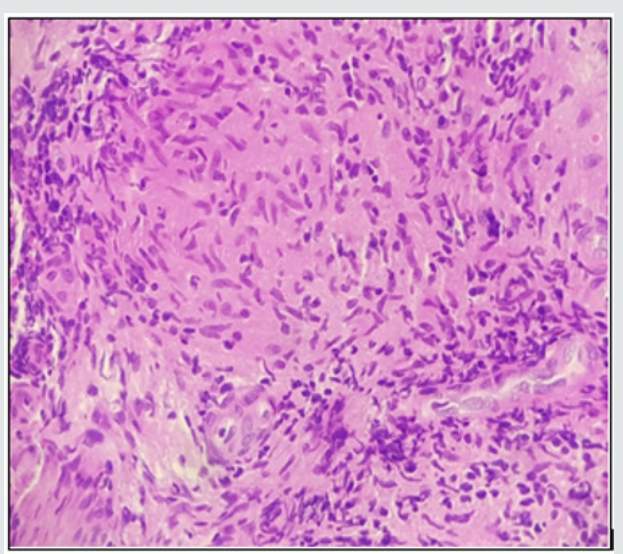

Figure 3: Biopsy of nail matrix (HE x 20)

Sarcoid granulomas in the dermis. 


\section{Discussion}

Sarcoidosis is a granulomatous systemic disease of undetermined etiology. Cutaneous manifestations are present in 25 to $35 \%$ of sarcoidosis and it is usually an early manifestation of the disease. On the other hand, nail changes are more uncommon and are often accompanied to systemic involvement. In a study released by Veien and colleagues $1.6 \%$ of patients with cutaneous sarcoidosis had a nail dystrophy [1]. Clinically, nail abnormalities may be in the form of nail dystrophy, pachyonychia, onycholysis, longitudinal striae, splinter hemorrhages, subungual hyperkeratosis, onychorrhexis, pterygium or squarely an anonychia $[2,3]$. In our case, we reported a new clinical appearances of nail sarcoidosis which is the hyperkeratotic verrucous lesions of the nail body and proximal nail fold and longitudinal nail fracture with localized nail plate deformity.

Dermoscopic features of nail sarcoidosis had never been described before. In our patient, there were dermoscopic signs in the proximal nail fold (pink coloration of the background, change of the microvascular architecture, linear vessels of different diameter, glomerular and serpiginous vessels, large areas of white scarlike depigmentation, translucent orange globular-like structures and white scale) and the nail plate (nail thickening, subungual hyperkeratosis, Splinter hemorrhages, dark dots, nonparallel lesion edg, Yellow or white nail plate and longitudinal parallel white lines). Ungueale involvement during sarcoidosis is often associated with digital bone cysts, dactylitis and lupus pernio, and in most cases is accompanied by cutaneous or systemic involvement (lymph node, pulmonary, articular or sinus involvement) [4,5]. Nail sarcoidosis treatment is based on oral, topical or intralesional corticosteroid therapy and synthetic antimalarials [6].

\section{Conclusion}

Nail involvement in sarcoidosis is unusual and is often associated with systemic involvement such as underlying bone location. the clinical aspects are multiple and can confuse with other nail diseases, hence the interest of the dermoscopy which can guide us in some cases.

\section{References}

1. Veien NK, Stahl D, Brodthagen H (1987) Cutaneous sarcoidosis in Caucasians. J Am Acad Dermatol 16(3): 534-540.

2. Momen SE, Al-Niaimi F (2013) Sarcoid and the nail: Review of the literature. Clin Exp Dermatol 38(2): 119-124.

3. Fernandez-Faith E, McDonnell J (2007) Cutaneous sarcoidosis: Differential diagnosis. Clin Dermatol 25(3): 276-287.

4. Baran R, Dawber RPR (1994) Diseases of the Nails and their Management ( $2^{\text {nd }}$ edn.) Blackwell Science, London, USA pp. 187-188.

5. Santoro F, Sloan SB (2009) Nail dystrophy and bony involvement in chronic sarcoidosis. J Am Acad Dermatol 60(6): 1050-1052.

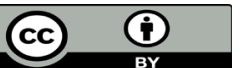

This work is licensed under Creative Commons Attribution 4.0 License

To Submit Your Article Click Here: Submit Article

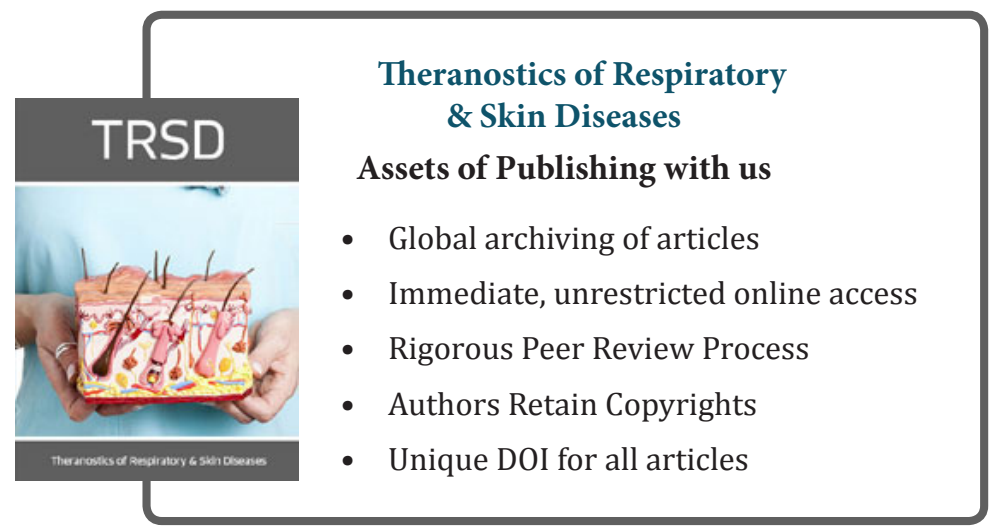

